\title{
Una relación potencialmente peligrosa: lupus eritematoso sistémico y enfermedad de Kikuchi - Fujimoto
}

\author{
A dangerous relation: Systemic lupus erythematosus disease and \\ Kikuchi Fujimoto disease
}

\author{
Juan Camilo Botero-Echeverri ${ }^{1 \otimes}$ CvLAC, Carlos Mauricio Muriel-Hurtado ${ }^{2}$, Ariel Antonio Arteta- \\ Cueto 3 CvLAC
}

Fecha correspondencia:

Recibido: mayo 24 de 2015.

Revisado: julio 29 de 2016.

Aceptado: septiembre 13 de 2016.

Forma de citar:

Botero-Echeverri JC, Muriel-Hurtado

CM, Arteta-Cueto AA. Una relación

potencialmente peligrosa: lupus

eritematoso sistémico y enfermedad

de Kikuchi - Fujimoto. Rev CES Med

2016; 30(2): 225-230.

\section{Open access}

(C) Copyright

Licencia creative commons

Ética de publicaciones

Revisión por pares

Gestión por Open Journal System

ISSN 0120-8705

e-ISSN 2215-9177

Sobre los autores:

1 Médico Internista-Epidemiólogo. Universidad CES - Clínica CES .

2 Residente de Medicina Interna Universidad CES.

3 Patólogo clínico. Universidad CES - Clínica CES.

Comparte

\section{Resumen}

La enfermedad de Kikuchi-Fujimoto (EKF) es conocida como una linfadenitis necrotizante histiocítica, tiene una presentación benigna y autolimitada, afecta principalmente a mujeres jóvenes y generalmente resuelve sin tratamiento en los primeros seis meses de los síntomas. Presentamos el caso de una mujer de 48 años quien consultó por un cuadro de dolor abdominal, mialgias y adenopatía cervical al examen físico. Se le encontró en los exámenes paraclínicos anticuerpos antinucleares 1:640, anti DNA de doble cadena asociado a consumo de complemento y anticuerpos anticardiolipina positivos. Se realizó biopsia de ganglio cervical que mostró necrosis cortical y población de histiocitos y linfocitos. Al iniciar el manejo con esteroides y cloroquina se comprobó mejoría franca y posteriormente la paciente fue dada de alta, continuando controles de manera ambulatoria por los servicios de reumatología y medicina interna.

Palabras clave: Enfermedad de Kikuchi-Fujimoto, Linfadenitis necrotizante, Lupus eritematoso sistémico.

\begin{abstract}
Kikuchi disease, also known as histiocytic necrotizing lymphadenitis, isa benign and self-limited condition that mainly affects young women andresolve without treatment within six months of symptoms. It was first describedin Japan in 1972 and is more common in Asian population.

We report the case of a woman of 48 years who consults for abdominal pain, myalgia, and cervical lymphadenopathy on physical examination. He found himself in the presence of antinuclear antibodies paraclinical 1: 640 positive DNA double chain associated with complement consumption and positive cardiolipin antibodies We perform cervical node biopsy that showed the presence of cortical necrosis and histiocytes and lymphocytes population. When you start handling steroid and chloroquine, a high frank and subsequent improvement was observed, continuing control rheumatology and internal medicine.
\end{abstract}

Keywords: Kikuchi-Fujimoto disease, Necrotising lymphadenitis, Systemic lupus erythematosus. 


\section{Introducción}

En la práctica clínica es habitual encontrar casos de pacientes que consultan a los servicios de urgencias por cuadros de fiebre de origen indeterminado asociados a la aparición de adenopatías. La presencia de esta sintomatología puede ser explicada por una amplia variedad de condiciones patológicas de etiología infecciosa, autoinmune, oncológica, hematológica, entre otras. Uno de los diagnósticos a considerar en pacientes con esta sintomatología es la enfermedad de Kikuchi-Fujimoto. El objetivo de este reporte de caso es informar a los lectores sobre una enfermedad poco común y alertar sobre sus potenciales complicaciones, entre las cuales incluso se encuentra la muerte.

Reportamos el caso de una mujer de 48 años con antecedentes personales de depresión y fibromialgia, quien consultó por fiebre y adenopatía cervical, y en quien se demostró una enfermedad de Kikuchi-Fujimoto.

Uno de los diagnósticos a considerar en pacientes con esta sintomatología es la enfermedad de Kikuchi-Fujimoto.

\section{Descripción del caso}

Mujer de 48 años, secretaria, quien consultó al servicio de urgencias por cuadro de siete días de evolución de mialgias, dolor abdominal tipo cólico, diarrea líquida no disentérica, náuseas y algunos episodios de emesis. También refería episodios de fiebre de predominio nocturno, asociados a diaforesis y dolores articulares en las manos. Reportaba antecedentes de trastorno depresivo y fibromialgia.

En el examen físico de ingreso se encontraba con presión arterial de 120/70 mm Hg. frecuencia cardiaca de 70 , frecuencia respiratoria de 16 y temperatura de $38{ }^{\circ} \mathrm{C}$. A nivel del cuello presentaba adenopatía única en región cervical anterior derecha de $3 \times 3 \mathrm{~cm}$; abdomen doloroso a la palpación profunda en flanco y fosa ilĺaca izquierda. Se realizaron algunos exámenes de laboratorio entre los que se encontraba un hemograma con leucopenia, neutropenia, linfopenia y un síndrome anémico de volúmenes normales; proteína $\mathrm{C}$ reactiva (PCR) y sedimentación elevadas, lactato deshidrogenasa (LDH) discretamente elevada.

Con la sospecha de enfermedad autoinmune se solicitó perfil de autoinmunidad con un factor reumatoide negativo, anticuerpos antinucleares (ANAS): 1:640 patrón homogéneo, complemento sérico con C3 consumido y C4 normal, ENAS negativo, anti DNA bicatenario positivo 1:160, anticuerpos anticardiolipina reactivos IgG: 24.6 GLP, IgM: 69.1 MLP.

Ante la persistencia del síndrome febril y la presencia de adenopatía única en el cuello se decide realizar biopsia excisional para descartar proceso linfoproliferativo asociado, la cual reportó: "linfadenopatía necrotizante tipo Kikuchi. Necrosis cortical. Acúmulos de histiocitos y linfocitos" (figuras 1 y 2 ).

Con la confirmación de enfermedad de Kikuchi - Fujimoto por biopsia de ganglio cervical y sobreposición con lupus eritematoso sistémico al cumplir criterios clínicos (dolor articular inflamatorio, leucopenia) y de laboratorio (ANAS, anti DNA, consumo de complemento, anti cardiolipinas), se decidió inició de tratamiento con prednisolona $30 \mathrm{mg} /$ día asociado a cloroquina $250 \mathrm{mg} /$ día.

Con este manejo la paciente presentó mejoría de sus dolores articulares, involución de la fiebre y mejoría de adenopatía cervical al cabo de 72 horas y finalmente pudo ser dada de alta en mejor condición general para continuar control ambulatorio por el servicio de reumatología. 


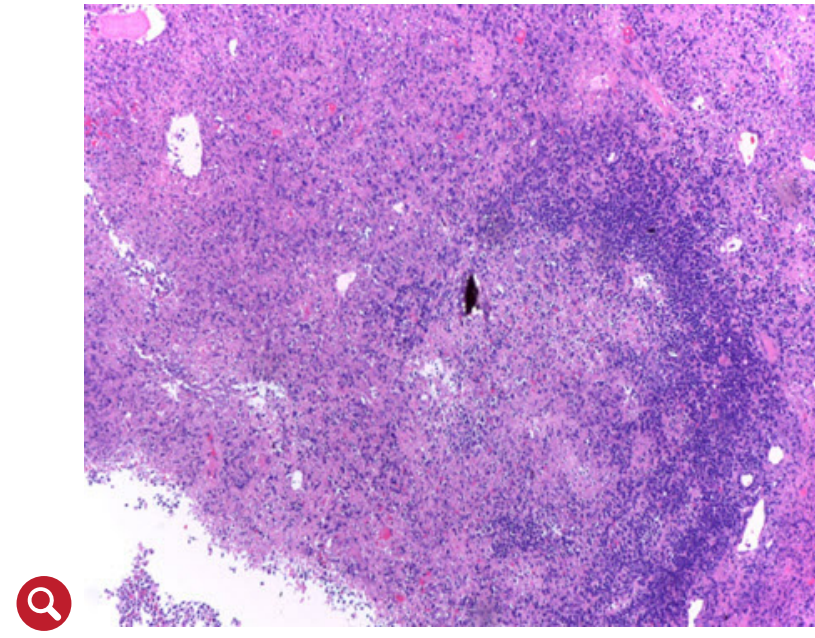

Figura 1. Presencia de histiocitos y linfocitos

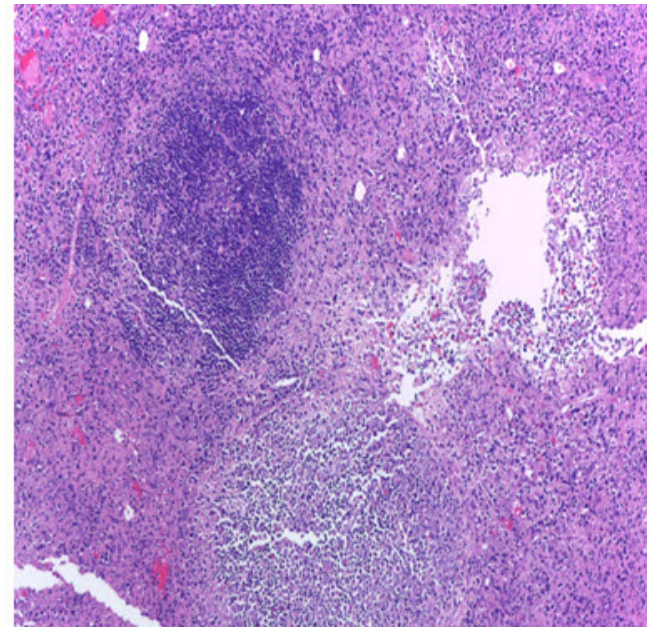

Figura 2. Histiocitos y necrosis cortical
La enfermedad Kikuchi-Fujimoto siempre debe ser un diagnóstico de exclusión por lo que deben ser descartadas otras causas: infecciosas autoinmunes, hematológicas, entre otras.

\section{Discusión}

La enfermedad de Kikuchi-Fujimoto, también conocida con el nombre de linfadenitis necrotizante histiocítica, es causa de síndrome febril de origen indeterminado asociado a linfadenopatías. Fue descrita por primera vez en 1972, se encuentra principalmente en personas asiáticas jóvenes y se caracteriza por la aparición de linfadenopatías generalmente menores a $1 \mathrm{~cm}$, lo cual las diferencia de procesos oncológicos en los que el tamaño habitual de las mismas es mayor (1).

Las características clínicas más importantes son fiebre, linfadenopatías de predominio cervical, leucopenia, síntomas respiratorios, artralgias, mialgias y manifestaciones de la piel (úlceras, placas eritematosas y pápulas) $(\underline{1}, \underline{3})$.

Antes de definir el diagnóstico se deben tener en cuenta una amplia variedad de diagnósticos diferenciales tales como enfermedad de Castleman o sarcoidosis. En la primera, el 80 \% de las adenopatías están ubicadas en el tórax, mientras que la sarcoidosis usualmente se caracteriza por la presencia de adenopatías hiliares o intratorácicas y manifestaciones respiratorias (1).

La enfermedad Kikuchi-Fujimoto siempre debe ser un diagnóstico de exclusión por lo que deben ser descartadas otras causas: infecciosas (citomegalovirus, mononucleosis infecciosa, toxoplasmosis, VIH, rubeola, tuberculosis ganglionar), autoinmunes (lupus eritematoso sistémico), hematológicas (linfomas), entre otras $(\underline{2}, \underline{4})$.

El diagnóstico de la entidad debe hacerse por biopsia excisional de adenopatías relacionadas. El patrón histológico característico revela histiocitos crescénticos, detritus cariorréxicos, escombros granulares y monocitos plasmocitoides (므).

La enfermedad puede autolimitarse a los seis meses de iniciada o tener una respuesta favorable al uso de esteroides. El pronóstico es generalmente bueno aunque puede presentar una mortalidad del $2 \%$ (1).

Para el manejo de los pacientes, además de los corticoesteroides, pueden ser útiles antibióticos como ciprofloxacina y minociclina; la hidroxicloroquina y los antinflamatorios no esteroideos (2). 
A pesar de su prevalencia predominante en la población asiática oriental, la enfermedad de Kikuchi-Fujimoto es cada vez más frecuente en poblaciones occidentales.
La asociación entre enfermedad autoinmunes, específicamente lupus eritematoso sistémico y Kikuchi-Fujimoto ha sido reportada en varios artículos a nivel mundial (ㅁ-요. . La enfermedad de Kikuchi-Fujimoto puede presentarse antes, después o superpuesta con el lupus eritematoso sistémico $(\underline{9}, \underline{10})$. También se ha encontrado relación con otras enfermedades autoinmunes como la enfermedad de Still, el síndrome de Sjögren, polimiositis y la artritis reumatoide (2).

En la revisión de Diez et al. se reporta el caso de una mujer de 22 años quien consulta por fiebre, adenopatía única cervical, con ANA's positivos 1:320 (patrón homogéneo) y biopsia del ganglio cervical que reporta necrosis paracortical, con detritus celulares y numerosos histiocitos, compatibles con linfadenitis necrotizante (ㄴ) , caso similar al reportado.

Ruaro y Sulli describen el caso de un hombre de 19 años de edad con cuadro clínico de adenopatías cervicales, poliartralgias, ANA's positivos de 1:1280 con patrón homogéneo. Se tomó biopsia de adenopatía cervical donde se confirma el diagnóstico de una enfermedad de Kikuchi-Fujimoto asociada a LES (2).

A pesar de su prevalencia predominante en la población asiática oriental, la enfermedad de Kikuchi-Fujimoto es cada vez más frecuente en poblaciones occidentales $(11,12)$. En Latinoamérica hay algunos reportes de caso de pacientes con la enfermedad, como lo descrito por Xavier et al., quienes reportan dos casos de mujeres con sospecha inicial de tuberculosis y posteriormente diagnosticadas con enfermedad de Kikuchi. En regiones endémicas con alta prevalencia de tuberculosis, este diagnóstico debe descartarse siempre antes de pensar en una enfermedad de Kikuchi-Fujimoto (11).

En Brasil (12) se reportan tres casos de pacientes de sexo femenino, no asiáticas, quienes consultan por cuadro de adenopatía cervical y posteriormente desarrollan fiebre; siendo luego llevadas a biopsia y obteniendo finalmente el diagnóstico de enfermedad de Kikuchi-Fujimoto. Estos casos son muy similares al reportado en este artículo, donde la paciente consultó inicialmente por similar sintomatología, aunque en esas pacientes no se demostró enfermedad autoinmune asociada.

En la búsqueda bibliográfica encontramos varios artículos donde se describen casos de enfermedad de Kikuchi-Fujimoto, en diversas poblaciones a nivel mundial, lo cual indica que aunque es una enfermedad infrecuente, debe sospecharse siempre en pacientes con cuadro febril de origen indeterminado y adenopatías de predominio cervical (13-15).

Aunque la enfermedad generalmente tiene buen pronóstico, también ha sido reportada con desenlaces fatales, como se describe en un artículo donde dos pacientes con diagnóstico de Kikuchi-Fujimoto y lupus fallecieron por la enfermedad (16).

\section{Conclusión}

La enfermedad de Kikuchi-Fujimoto en superposición con lupus eritematoso sistémico es un diagnóstico a tener en cuenta en pacientes que consultan al servicio de urgencias por dicha sintomatología.

\section{Conflicto de intereses}

Los autores declaran que no tienen conflicto de interés 


\section{Bibliografía}

1. Ray A, Muse VV, Boyer DF. Case 38-2013: A 30-Year-Old Man with Fever and Lymphadenopathy. N Engl J Med. 2013; 369(24): 2333-43 https://www.ncbi.nlm.nih. gov/pubmed/24328468

2. Ruaro B, Sulli A, Alessandri E, Fraternali- Orcioni G, Cutolo M. Kikuchi-Fujimoto's disease associated with systemic lupus erythematous: difficult case report and literature review. Lupus 2014; 23: 939-44. https://www.ncbi.nlm.nih.gov/pubmed/24739458

3. Dumas G, Prendki V, Haroche J, Amoura Z, Cacoub P, Galicier L et al. Kikuchi-Fujimoto disease retrospective study of 91 cases and review of the literature. Medicine (Baltimore). 2014; 93(24):372-82. https://www.ncbi.nlm.nih.gov/pubmed/25500707

4. Diez - Morrondo C, Pantoja-Zarza L, Manjón -Haces JA. Kikuchi Fujimoto's disease appearing as systemic lupus erythematosus. Reumatol Clin. 2012; 8(3):15355. https://www.ncbi.nlm.nih.gov/pmc/articles/PMC4935008/

5. Weiss LM, O'Malley D. Benign lymphadenopathies. Modern Pathology 2013; 26: S88-S96. http://www.nature.com/modpathol/journal/v26/n1s/full/modpatho$\underline{\text { l2012176a.html }}$

6. Cramer J, Schmiedel S, Alegre NG, Schafer H, Burchard GD, Merz H. Necrotizing lymphadenitis: Kikuchi-Fujimoto disease alias lupus lymphadenitis?. Lupus (2010); 19:89-92. https://www.ncbi.nlm.nih.gov/pubmed/19933723

7. Gionanlis L ,Katsounaros M , Bamihas G, Fragidis S, Veneti P, Sombolos K. Kikuchi-Fujimoto disease and Systemic Lupus Erythematosus: The EBV connection? Renal Failure. 2009; (31)2:144-8. https://www.ncbi.nlm.nih.gov/pubmed/19212912

8. Kim SK, Kang SM, Yoon BY, Kim DY, Cho SK. Histiocytic necrotizing lymphadenitis in the context of systemic lupus erythematosus (SLE): Is histiocytic necrotizing lymphadenitis in SLE associated with skin lesions? Lupus. 2011; 20 (8): 809-19. https://www.ncbi.nlm.nih.gov/pubmed/21562017

9. Lamzaf, L, Harmouche H, Maamar M, Adnaoui M, Aouni M, Tazi Mezalek Z. Kikuchi-Fujimoto disease: Report of 4 cases and review of the literature. European Annals of Otorhinolaryngology, Head and Neck diseases. 2014; 131 (6):329-32. https://www.ncbi.nlm.nih.gov/pubmed/25294358

10. Baltaci M, Fritsch P. Histologic features of cutaneous lupus erythematosus. Autoimmunity Reviews. 2009; 8(6):467-73. https://www.ncbi.nlm.nih.gov/pubmed/19162243

11. Xavier, RG, Silva DR, Keiserman MW, Lopes MF . Kikuchi-Fujimoto disease. J Bras Pneumol. 2008; 34(12):1074-78. https://www.ncbi.nlm.nih.gov/pubmed/19180344

12. K Sousa Ade A, Soares JM, Sá Santos MH, Martins MP, Salles JM. Kikuchi-Fujimoto disease: three case reports. Sao Paulo Med J. 2010; 128 (4): 232-5. https:// www.ncbi.nlm.nih.gov/pubmed/21120436 
13. Tariq H, Gaduputi V, Rafiq A, Shenoy R.The enigmatic Kikuchi-Fujimoto disease: a case report and review. Case Reports in Hematology. 2014; 2014, 648136. https:// www.hindawi.com/journals/crihem/2014/648136/

14. Akinbami A, Odesanya M, Soyemi S, John-Olabode S, Adediran A, Oshinaike 0 et al. The Kikuchi-Fujimoto disease in Nigeria: a case report and literature review. Case Reports in Medicine. 2014; 2014:171029. https://www.ncbi.nlm.nih.gov/pmc/ articles/PMC4020498/

15. Aota N, Hirahara K, Kano Y, Fukuoka T, Yamada A, Shiohara T Systemic Lupus Erythematosus presenting with Kikuchi-Fujimoto's disease as a longterm sequela of drug-induced hypersensitivity syndrome. Dermatology. 2009; 218(3):275-7. https://www.ncbi.nlm.nih.gov/pubmed/19088463

16. Sharma V, Rankin R. Fatal Kikuchi-like lymphadenitis associated with connective tissue disease: a report of two cases and review of the literature. Sharma and Rankin Springer Plus. 2015; 4:167. https://springerplus.springeropen.com/articles/10.1186/s40064-015-0925-7 